\title{
EDITORIAL
}

\section{Invasive assessment of myocardial bridges}

\section{J Lovell, C J Knight}

\begin{abstract}
Myocardial bridges may cause clinically relevant problems only in certain patients. Therefore, methods of assessment which can identify those bridges that may cause ischaemia are required in order to guide treatment
\end{abstract}

See end of article for authors' affiliations

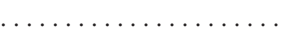

Correspondence to: Dr C J Knight, London Chest Hospital, Bonner Road, London E2 9JX, UK; knightlch@aol.com
It has been recognised for over 200 years that epicardial coronary arteries may be crossed by muscular bands for limited segments of their courses. ${ }^{1}$ The term myocardial bridge was first employed in 1961 in a case report describing angiographic systolic narrowing. ${ }^{2}$ Opinion remains divided as to whether myocardial bridges have pathological consequences or are merely epiphenomena. The clinical consequences of myocardial bridges are difficult to evaluate and invoking the presence of a myocardial bridge as a cause of myocardial ischaemia remains not wholly respectable in the view of many cardiologists. On the one hand a wide range of clinical problems, including acute coronary syndromes and arrhythmias, have been reported in patients whose sole apparent cardiac abnormality is the presence of a myocardial bridge. ${ }^{23}$ Conversely, myocardial bridges may be identified in asymptomatic individuals, there seems little correlation between the severity of systolic narrowing and clinical outcome, and doubts remain about the pathophysiological relevance of a purely transient systolic narrowing of a coronary artery when the predominant phase of coronary blood flow occurs in diastole. The answer to this conundrum is likely to be that at some times, in some patients, some muscle bridges may cause clinically relevant problems. What is required to guide therapy are methods of assessment to identify those bridges that may cause ischaemia.

The last decade has seen advances in invasive technology that have improved the ability to measure both anatomical and physiological parameters of coronary vessels. The use of these techniques in patients with muscle bridges has improved our understanding of the pathophysiology of this interesting condition.

\section{ANGIOGRAPHY}

Quantitative measurements of vessel diameter throughout the cardiac cycle are now possible following the introduction of digitised angiography. Measurements in patients with myocardial bridges have revealed a persistent diastolic diameter reduction enduring to mid diastole. ${ }^{4-6}$ The largest study of 42 patients documented a mean maximum systolic reduction of $71 \%$ with a persistent reduction of 35\% during mid diastole, $12 \%$ of patients having $>50 \%$ reduction in mid diastole.

\section{INTRAVASCULAR ULTRASOUND}

Results from studies using intravascular ultrasound (IVUS) have complemented the findings from quantitative angiographic assessments and confirmed systolic compression of the bridged segment with delayed relaxation and reduction of vessel lumen diameter in diastole. ${ }^{7}$ The systolic compression is usually eccentric rather than concentric. ${ }^{78}$ IVUS studies have also consistently shown a high incidence of atherosclerotic plaques in the coronary artery segment immediately proximal to the myocardial bridge. Ge and colleagues in 1994 and 1999 found 12 of 14 (86\%) and 61 of $69(88 \%)$ patients respectively with atherosclerotic involvement of the proximal segment. ${ }^{7}$ In the second study the mean area stenosis was $42 \%$ and no plaques were found in the bridge or distal segment. None of the proximal lesions detected by IVUS in the initial study had been seen on angiography. The superior sensitivity of IVUS in identifying atherosclerotic plaques in patients with myocardial bridging has also been demonstrated in case studies. ${ }^{9} 10$

A specific IVUS sign for myocardial bridging has also been noted, which is an echolucent halfmoon seen between epicardial tissue and the coronary artery throughout the cardiac cycle but not in a normal segment. ${ }^{8}$ This appearance was seen in all patients studied with IVUS and was localised to the bridged segment.

\section{INTRACORONARY DOPPLER}

Introduction of intracoronary flow velocity and pressure measurement has allowed detailed investigation of the haemodynamic changes associated with myocardial bridging. Consistent characteristic changes in intracoronary flow have been reported using 0.0014 inch Doppler wires measuring coronary flow velocity throughout the cardiac cycle, proximal and distal to the myocardial bridge. The most striking finding from these studies is an abnormal Doppler flow profile with an abrupt early diastolic flow acceleration and rapid mid diastolic deceleration followed by a mid to late diastolic plateau in the bridged segment. This has been described as a spike and dome appearance or fingertip phenomenon. ${ }^{38}$ These changes represent increased blood flow, generated in diastole by a rapid lowering in resistance of the distal coronary vessels. This blood flow meets the still compressed bridged segment producing the early Doppler peak. As the lumen increases, a mid diastolic drop precedes the plateau reached at maximal vessel size. These changes are somewhat analogous to the systolic profile seen in hypertrophic obstructive cardiomyopathy caused by dynamic obstruction generated by septal hypertrophy and systolic anterior motion of the mitral apparatus. In myocardial 
bridging, however, the Doppler flow pattern in systole illustrates reduced or even absent antegrade systolic flow and in some cases retrograde flow in late systole. ${ }^{811}$ The functional significance of these findings is confirmed by reduced coronary flow reserve measured in the vessel distal to the bridge. This is assessed with the aid of intracoronary adenosine or papaverine to produce a hyperaemic reaction, usually expressed as a ratio of hyperaemic to basal flow. Coronary flow reserve is usually $>3$ in a normal study but the bridged segments are returning values consistently below this. ${ }^{4-811} 12$

\section{INTRACORONARY PRESSURE}

Results of intracoronary pressure measurement have given contradictory results in patients with myocardial bridges. Two patients have shown a significant pressure fall across the bridged segment and both had reduced coronary fractional flow reserves $<0.75,{ }^{11}{ }^{13}$ a threshold which has been extensively validated in patients with coronary artery disease ${ }^{14}$ However, these results were not replicated in a larger study of 12 patients in which the only abnormal pressure change was a significantly higher peak systolic pressure within the bridged segment compared to either side. No pressure gradient was demonstrated across the bridged segment. ${ }^{6}$

\section{CONCLUSION}

The limited information that we have on the outcome of patients with myocardial bridges indicates that the risk of serious clinical consequences is low. In one study of 28 patients, followed up for an average of 11 years, none experienced a myocardial infarct. ${ }^{15}$ Given this excellent prognosis it seems clear that asymptomatic patients should not be treated. Patients in the above studies represent a highly selected subset of patients in that all were experiencing symptoms and most had objective markers of myocardial ischaemia. The evidence from these patients suggests that myocardial bridges may affect both systole and diastole and produce significant haemodynamic changes in coronary flow. It seems likely that myocardial bridges can, therefore, have a pathological role in producing ischaemia in some patients. Within this subset of patients there is also a high incidence of associated atheroma.

For the patient with typical symptoms and evidence of myocardial ischaemia the first line treatment should be a $\beta$ blocker as there is evidence of symptomatic improvement, reduction in narrowing, and resolution of Doppler flow patterns following $\beta$ blockade. ${ }^{4}$ A small subset of patients may have persistent symptoms despite such treatment and should be investigated further. IVUS, pressure, and flow wire studies are recommended to provide the necessary data for the most appropriate management decision. IVUS is especially useful in excluding proximal atherosclerotic plaques that may be missed on angiography. If these studies fail to show any significant haemodynamic changes, then the patient and cardiologist can be reassured and conservative management continued. In the event of significant positive results, interventional or surgical treatment could be considered. Treatment for myocardial bridging with myotomy, bypass grafts or coronary stents have all been reported to abate symptoms and normalise non-invasive stress tests and haemodynamic abnormalities. ${ }^{35121316}$ Surgical or percutaneous intervention for intractable symptoms should not be performed in this benign condition without detailed prior haemodynamic and IVUS assessment.

\section{Authors' affiliations}

M J Lovell, C J Knight, London Chest Hospital, London, UK

\section{REFERENCES}

1 Yamaguchi M, Tangkawattana P, Hamlin RL. Myocardial bridging as a factor in heart disorders: critical review and hypothesis. Acta Anat 1996; 157:248-60.

2 Angelini $\mathbf{P}$, Tivellato $M$, Donis J, et al. Myocardial bridges: a review. Prog Cardiovasc Dis 1983;26:75-88.

3 Tio RA, Van Gelder IC, Boonstra PW, et al. Myocardial bridging in a survivor of sudden cardiac near-death: role of intracoronary Doppler flow measurements and angiography during dobutamine stress in the clinical evaluation. Heart 1997;77:280-2.

4 Schwarz ER, Klues HG, vom Dahl J, et al. Functional, angiographic and intracoronary Doppler flow characteristics in symptomatic patients with myocardial bridging: effect of short-term intravenous beta-blocker medication. J Am Coll Cardiol 1996;27:1637-45.

5 Schwarz ER, Klues HG, vom Dahl J, et al. Functional characteristics of myocardial bridging - a combined angiographic and intracoronary Doppler flow study. Eur Heart J 1997; 18:434-42.

6 Klues HG, Schwarz ER, vom Dahl J, et al. Disturbed intracoronary haemodynamics in myocardial bridging. Early normalization by intracoronary stent placement. Circulation 1997;96:2905-13.

7 Ge J, Erbel R, Rupprecht HJ, et al. Comparison of intravascular ultrasound and angiography in the assessment of myocardial bridging. Circulation 1994:89:1725-32.

8 Ge J, Jeremias A, Rupp A, et al. New signs characteristic of myocardial bridging demonstrated by intracoronary ultrasound and Doppler. Eur Heart J 1999;20:1707-16.

9 Kneale BJ, Stewart AJ, Coltart DJ. A case of myocardial bridging: evaluation using intracoronary ultrasound, Doppler flow measurement, and quantitative coronary angiography. Heart 1996;76:374-6.

10 Winter RJ, Kok WEM, Piek JJ. Coronary atherosclerosis within a myocardial bridge, not a benign condition. Heart 1998;80:91-3.

11 Ge J, Erbel R, Gorge G, et al. High wall shear stress proximal to myocardial bridging and atherosclerosis: intracoronary ultrasound and pressure measurements. $\mathrm{Br}$ Heart J 1995:73:462-5.

12 Hagger PK, Schwarz ER, vom Dahl J, et al. Long term angiographic and clinical follow up in patients with stent implantation for symptomatic myocardial bridging. Heart 2000;84:403-8.

13 Prendergast BD, Kerr F, Starkey RI. Normalisation of abnormal coronary fractional flow reserve associated with myocardial bridging using an intracoronary stent. Heart 2000:83.705-7.

14 Pijls NH, Bruyne BD, Peels K, et al. Measurement of fractional flow reserve to assess the functional severity of coronary artery stenoses. N Engl J Med 1996;334:1703-8.

15 Juillière $Y$, Berder $V$, Suty-Selton $C$, et al. Isolated myocardial bridges with angiographic milking of the left anterior descending coronary artery: a long-term follow-up study. Am Heart J 1995:129:663-5.

16 Roul G, Sens P, Germain P, et al. Myocardial bridging as a cause of acute transient left heart dysfunction. Chest 1999; 1 16:574-80. 